\title{
After propensity score matching in long-term oncologic outcomes of robotic gastrectomy for gastric cancer compared with laparoscopic gastrectomy
}

\author{
Jong Won $\mathrm{Kim}^{1}$ (D)
}

Received: 30 May 2018 / Accepted: 13 June 2018 / Published online: 16 June 2018

(c) The International Gastric Cancer Association and The Japanese Gastric Cancer Association 2018

I recently read interestingly "Long-term oncologic outcomes of robotic gastrectomy for gastric cancer compared with laparoscopic gastrectomy" [1]. The authors compared robotic gastrectomy with laparoscopic gastrectomy for gastric cancer, and it is thought that the results are very meaningful because there are many cases and long follow-up period. Although many cases have been included, they have tried to reduce the bias by performing propensity score matching (PSM) considering that this is a retrospective data collection study. However, while reading the paper interestingly, I found some strange points in the data presented in the paper.

There are several ways to implement PSM. However, basically, a case with similar characteristics to each case of the experimental group (usually group which has a smaller number of cases between two groups) is selected from the control group. Therefore when PSM is performed, the number of cases is equal or less after implementation [2]. However, in Table 1, in the cT classification, the number of cases in Robotic group is increased in T1 cases. Also, in $\mathrm{cN}$ classification, it can be seen that the number of cases of N0 increased after PSM in Robotic group. Also, in cStage, the number of cases of I increases after PSM in Robotic group. In the section describing the PSM method, it was described that the clinical stage is one of the variables considered when matching. Therefore, I tried to confirm that there was no difference in clinical stage between two groups after PSM, but the figure is not understood. It is necessary to confirm whether the result is a simple typing error or reevaluation of clinical stage after PSM.

After PSM, the number of case in Robot gastrectomy group decreased from 315 to 311 , and the metastatic LN

Jong Won Kim

drholy@gmail.com

1 Department of Surgery, Chung-Ang University Hospital, Chung-Ang University College of Medicine, 102, Heukseok-ro, Dongjak-gu, Seoul 06973, South Korea number decreased from $1.0 \pm 3.1$ (mean \pm standard deviation) to $0.6 \pm 2.4$. However, the cases excluded after PSM were 3 in N0 and 1 in N1. If so, at most the metastatic LN sum is reduced by 2 , so the mean value cannot be reduced by 0.4 . Considering the rounding off to the nearest integer at the second decimal place, I consider the scenario to be reduced to the smallest difference as follows. Mean value would be reduced from 0.950 to 0.649 and the sum would be reduced from $299.25(=0.950 \times 315)$ to $201.439(=0.649 \times 311)$, which means that there would be a decrease in at least 98 (= 299-201) metastatic LN in sum after PSM. However, as mentioned above, the maximum decrease after PSM would be just 2 . Therefore, I cannot help but think that there is an error in the number.

It is presumed to be a simple expression error and it is not a fatal error because it has no such close relation to the subject of the article. However, I think it is necessary to confirm because it can confuse the readers.

\section{Compliance with ethical standards}

Conflict of interest The authors declare that they have no conflict of interest.

Ethical standards This is not a study involving human or animal subjects, but just a response to published article.

\section{References}

1. Obama K, Kim YM, Kang DR, Son T, Kim HI, Noh SH, et al. Long-term oncologic outcomes of robotic gastrectomy for gastric cancer compared with laparoscopic gastrectomy. Gastric Cancer. 2018;21(2):285-95. https://doi.org/10.1007/s10120-017-0740-7 (Epub 2017/06/24).

2. Austin PC. An introduction to Propensity score methods for reducing the effects of confounding in observational studies. Multivar Behav Res. 2011;46(3):399-424. https://doi.org/10.1080/00273 171.2011.568786 (Epub 2011/08/06). 